\title{
The Impact and Outcome of Training Programme of Health Care Workers with Reference to Sample Collection in Microbiology Laboratory
}

\author{
Ruby Naz' , Naveen Saxena², Sameena Khan ${ }^{3}$ \\ 1, 2, Department of Microbiology, Government Medical College, Kota, Rajasthan, India. \\ ${ }^{3}$ Department of Microbiology, DY Patil Medical College and Hospital and Research Pune, India.
}

\section{ABSTRACT}

\section{BACKGROUND}

Sample collection of blood, urine, stool, sputum and swab collection for various tests in a microbiology laboratory is a very important and crucial part of laboratory investigations. All samples should be properly labelled, and the requisition form should be filled properly. The purpose of this study was to determine the effect of the training programme to improve the quality of sample collection in the microbiology laboratory.

\section{METHODS}

It is an observation based cross-sectional study which was conducted among the health care workers of a tertiary care hospital in Rajasthan to assess their knowledge regarding safe and proper sample collection and transportation.

\section{RESULTS}

In pre-session a lot of loopholes were found among the healthcare workers regarding proper sample collection techniques, handling of sample, transportation and storage of the sample, dealing with blood spillage and in general disinfection. After the orientation session, there was a significant increase in knowledge of how \& when to take a sample that is $63 \% \& 54 \%$ respectively which is almost more than double presession.

\section{CONCLUSIONS}

Proper sample collection and transportation are key steps to patient care and very useful for correct diagnosis. Most of the errors happen in the pre-analytical phase. In our study, we observed that the knowledge of participants improved significantly after continuous training sessions. This indicates that such a type of knowledge session is essential for improving sample collection practices. It is therefore important to train health care workers (HCW), who form the root of the health care system at regular intervals, to enhance knowledge significantly.

\section{KEY WORDS}

Sample Collection, Training, Laboratory, Sample Collection
Corresponding Author: Dr. Ruby Naz,

\# 49, R K Nagar Police Line, Baran Road, Kota-324001,

Rajasthan, India.

E-mail: drrubynaz@gmail.com

DOI: $10.14260 / j e m d s / 2021 / 657$

How to Cite This Article:

Naz R, Saxena N, Khan . The effect and outcome of training programme of health care workers with reference to sample collection in microbiology laboratory. J Evolution Med Dent Sci 2021;10(37):32373241, DOI: 10.14260/jemds/2021/657

Submission 18-02-2021,

Peer Review 29-07-2021,

Acceptance 06-08-2021,

Published 13-09-2021.

Copyright (C) 2021 Ruby Naz et al. This is an open access article distributed under Creative Commons Attribution License [Attribution 4.0 International (CC BY 4.0)] 


\section{BACKGROUND}

Microbiological laboratory test results are very important for clinicians to make a correct diagnosis of the patient's illness. Sample collection is a crucial part of these investigations. ${ }^{1}$ It is also important that all specimens should be properly labelled and requisition forms should be properly filled. ${ }^{2}$

Improperly collected samples with poor transportation systems may lead to a major error in diagnostic investigations. Most commonly these mistakes are at the pre-analytical level which can be easily preventable by proper training and monitoring. 3,4

Health care workers should be well aware and updated about collecting samples properly. In the microbiology laboratory, these improperly collected specimens fail to isolate causative organisms and recovery of contaminants or normal microbiota.5,6 The purpose of this study was to determine the effect of the training programme to improve the quality of sample collection in the microbiology laboratory.

\section{METHODS}

It is a descriptive study that was conducted among the health care workers of a tertiary care hospital in Rajasthan to assess their knowledge regarding safe and proper sample collection and transportation.

The study population comprised resident doctors, staff nurses and laboratory technicians from various departments including microbiology, medicine, paediatrics, surgery, orthopaedic and obstetrics and gynaecology. A total of 200 health care workers gave consent and participated in this study from February 2019 to January 2020. We designed a pre-session questionnaire before training and a post-session questionnaire after the full training program was completed to enquire their knowledge regarding the collection of samples, its timing, storage, transportation and report turnover time. Most of the health care workers are already skilful but sometimes without an update of knowledge they start to make mistakes. This method is based on a study conducted by Tenney J H et al. previously. ${ }^{7}$

Firstly, we want to know about their knowledge about proper sample collection for which we give them a questionnaire about sample collection. This questionnaire included questions regarding time and amount of samples like urine, blood, CSF and stool collection and transportation. Consent was taken from participants to take part in this study. The mean time for completing the questionnaire was 10 minutes. The question paper was confidential to participants and privacy was strictly maintained during this study. A knowledge session of one hour to enhance their knowledge in above mentioned all aspects was taken. This session included knowledge about proper time of blood, CSF and urine collection, appropriate amount according to age and proper guidelines related to the transport of sample collection.

A post-session questionnaire with the same questions was distributed and their knowledge after the session was checked. We held a total of 31 sessions in one year and did a descriptive analysis of one-year data. ${ }^{8,9}$
RESULTS

In pre-session, a lot of loopholes were found among the healthcare workers regarding proper sample collection techniques, handling of sample, transportation and storage of the sample, dealing with blood spillage and in general disinfection.

About $33 \%$ of health care workers had knowledge regarding proper ways to collect samples. Only $23 \%$ of HCWs knew when to collect the sample. $34 \%$ of HCWs had the idea of how to store samples and about $21 \%$ regarding their transportation. Only $28 \%$ of HCWs knew when to expect a report of a test or the time taken to process the samples. About $38 \%$ of HCWs had knowledge about how to take a sample when a patient was suspected of having tuberculosis. And about $42 \%$ of HCWs had a general idea of what to do in case of blood spillage and disinfection.

Many health workers didn't know how much volume of blood was sufficient to be inoculated in a blood culture bottle as we often received inadequate quantities of blood samples. Only $22 \%$ agreed that 1-2 ml blood should be inoculated in a $10 \mathrm{ml}$ culture in case of a child and most were in favour of 5-6 $\mathrm{ml}$ blood. The volume of blood to be inoculated per culture is the most important variable which can affect the recovery of microorganisms from a patient's sample with sepsis. There are so many studies that confirmed the more the volume cultured, the higher the rate of detection of bloodstream infection, reporting an increase in yield from $0.7 \%$ to $4.7 \%$ per extra millilitre of blood cultured. However, to prevent the inhibitors present in the blood, for example, host serum factors, complements, defensins, lysozymes, and other antimicrobial agents which inhibit the growth of bacteria in culture, 1-2 ml blood should be inoculated in a bottle with $10 \mathrm{ml}$ media. ${ }^{10,11}$

Therefore, inoculated blood must be diluted to minimise the effect of inhibitory substances. The recommended dilution factor is up to 10 times. $^{6}$ Time-to-time educational interventions like training and seminars are necessary for the study area to bridge the knowledge gap so that the quality of blood culture should improve. ${ }^{10}$

When the questionnaire was about the time to take blood for culture, $57 \%$ gave answers as only when the patient was febrile $>38^{\circ} \mathrm{C}$, with or without sepsis, $34 \%$ when the patient felt rigours and $10 \%$ at the time of admission. ${ }^{11}$

The literature indicates that blood culture has to be taken only when there is a critical indication to do so. After identification of signs and symptoms of bacteraemia or sepsis and before administration of antibiotics. ${ }^{10}$

If antibiotics are continued then blood should be taken just before administration of the next dose, with the exception of paediatric patients. Blood should be taken by trained people. Literature also indicates that blood should not be repeated by 2-5 days. The use of this blood culture surveillance had been used only in those patients who were waiting for organ transplantation. ${ }^{8}$

Literature also indicates about the site of collection, wherein peripheral venipuncture is the preferred site. To collect the blood drawn from the intravascular site is not optimal for they can be contaminated by organisms colonizing the hub or the wall of the catheter. ${ }^{11,12}$

In our questionnaire, $68 \%$ answered that we can collect blood from both site venipuncture and intravascular catheter. In the post-session questionnaire, $73 \%$ of people understood 
about the right volume of blood, $55 \%$ about the indication of blood culture and, and $88 \%$ gave answers that venipuncture should be preferred for sample collection for blood culture.

In response to a questionnaire about wearing sterile gloves during venipuncture, $60 \%$ responded of wearing sterile gloves, $12 \%$ didn't wear them at all and the rest wore only when re-palpating the vein after skin decontamination. After the session, $78 \%$ agreed to use gloves for the whole-time during blood collection. The literature said that a person drawing the blood should not palpate the vein after skin disinfection unless sterile gloves are worn. To avoid crosscontamination, it is fundamental not to re-palpate the vein. ${ }^{13}$ 14 There are so many discrepancies about the use of skin decontaminants before venipuncture. $43 \%$ use spirit, $23 \%$ use povidone-iodine and the rest use chlorhexidine.

Literature said that before venipuncture skin preparation should be done carefully with the use of alcohol or alcoholic chlorhexidine or tincture of iodine rather than povidoneiodine. Should allow adequate time for skin contact and let it dry. ${ }^{15,11}$ Alcoholic product is better in reduction of blood culture contamination, povidone iodine may cause skin irritation, alcoholic chlorhexidine needs 15 to 30 seconds to dry and tincture iodine 30 seconds and povidone-iodine needs 2 minutes to dry. Alcoholic chlorhexidine is best for skin decontamination and degreasing. ${ }^{16}$ In the present study, $83 \%$ gave answers about using alcohol for decontamination of skin.

CSF culture is essential in every meningitis patient but awareness was much below expectation. In this study, knowledge regarding a delay in transportation of cerebrospinal fluid, only $32 \%$ of participants answered that it should be stored in the refrigerator, $42 \%$ agreed with storing the specimen at room temperature while rest were in favour that it should not be stored in incubator. ${ }^{16}$ Literature said CSF should not be stored at low temperatures in the refrigerator or at high temperatures in sunlight. It should be transported immediately as soon as possible. If delayed, must be kept at room temperature for a short time. ${ }^{17}$ In the post-session questionnaire only $68 \%$ agreed that samples should be sent immediately to the laboratory.

The final question was about the timing of collection of stool samples and urine samples with microbiological swabs. $55 \%$ took swabs only as indicated, $45 \%$ performed microbiological swabs as a routine procedure for hospital admission, $22 \%$ performed routine swabs weekly from all the admission in hospital, and rest of them took swabs after the first spike of fever. In the case of a urine sample for culture and sensitivity, $37 \%$ performed urine culture only in those cases where patients reported some clinical symptoms or signs of cystitis, $44 \%$ performed urine culture at the time of hospital admission, $14 \%$ of culture samples were performed only in catheterised patients. ${ }^{17,18}$ In the case of stool samples, $66 \%$ took stool culture samples only in cases of diarrhoea, $19 \%$ took samples during hospital admission, $12 \%$ performed weekly samples from the time of admission in hospital.

The literature suggests that routine fungal or bacterial culture should not be done in asymptomatic patients. The site of culture should be decided according to signs and symptoms. After the session, only $23 \%$ of resident doctors gave answers that microbiological culture should be taken from symptomatic patients. ${ }^{19,10}$ This part we have to work more in our future training session. Still, physicians send samples without signs and symptoms.
After the orientation session, there was a significant increase in knowledge of how \& when to take samples that is $63 \%$ \& $54 \%$ respectively which is almost more than double the pre-session. Regarding the storage of samples, almost 76 $\%$ gave the correct answer. There was 3 times increase in knowledge of how to transport samples and $71 \%$ and $76 \%$ of people knew the processing of tuberculosis samples. The knowledge of blood spillage handling also increased up to double from pre-session. Overall training doubled the knowledge of HCW.

\begin{tabular}{|ccc|}
\hline When to collect blood sample & $\begin{array}{c}\text { Test Results in \% } \\
\text { before Session }\end{array}$ & $\begin{array}{c}\text { Test Result } \\
\text { after Session }\end{array}$ \\
How to store CSF & 23 & 63 \\
What to do in case of blood spillage & 34 & 68 \\
Site of blood collection & 38 & 81 \\
How to disinfect during blood collection & 55 & 73 \\
How to transport urine sample & 43 & 83 \\
\hline Table 1. Comparison of Both Results before and after Session. \\
\hline
\end{tabular}

\section{DISCUSSION}

Specimen collection and transportation play a major role in timely reporting and appropriate results of the investigation which was required for diagnosis. Health care workers play a crucial role in providing first-hand bedside care and their action has a direct role in the positive patient outcomes as it affects sample quality which is processed in the laboratory. It is therefore important to arrange regular training sessions to enhance knowledge, improve their attitude regarding proper sample collection, storage and transportation. ${ }^{20,11}$

In the present study, $57 \%$ were males and $43 \%$ were females and $29 \%$ were resident doctors, $54 \%$ were staff nurses and $17 \%$ were laboratory technicians. Of all participants $43 \%$ had less than 5 years of experience, $25 \%$ were between 5-10 years, and the rest had more than 15 years of experience. While the study was done by Chandak, Poonam et al. $35 \%$ had up to 5 years experience, $49 \%$ had 6-10 years' experience and $10 \%$ had 11 - 15 years experience. ${ }^{9}$ In our study, the knowledge regarding how \& when to collect the sample, storage of the sample, when to expect a report, how to transport sample and processing of sample was increased by double while a study done by B. Sandeep et al. ${ }^{21}$ showed the increase of knowledge by only $32 \%$. In our study, the knowledge regarding blood spillage handling increased by 81 $\%$ while another study showed the increase of knowledge by $30 \% .{ }^{21}$

The reason for the lower performance on these questions about sample collection in the pre-session questionnaire may be that most doctors, apart from the laboratory physicians, do not have sufficient knowledge of the processes in the laboratory. ${ }^{22}$

Once the specimens are submitted to the laboratories, they are ignorant of the further procedures done on those samples. It is an important step to increase knowledge about sample collection that all doctors, especially residents, are made to rotate through the laboratories to acquaint themselves with proper sample collection. Some researchers suggest the importance of rotatory postings of clinical residents in laboratories to acquaint them with the importance of proper collection and transport of specimens. ${ }^{11}$ 
The literature tells us that fungal cultures should not be performed in those patients who are asymptomatic. All the bacterial and viral cultures from sites of suspected infection should be performed only if it is indicated. ${ }^{16,17}$

Some clinicians propose these viral fungal investigations as routine due to the lack of proper reference documents. Another factor is possibly the limited number of CME or training schedules. The literature always emphasizes the diagnostic importance of microbiological cultures, and hence the importance of how the sample is collected. Samples can be contaminated by many sources such as the material used to collect samples, the patient's skin and the hands of the person taking the sample or vial or container contamination. Sometimes delay in sample transport is also an important reason for false reporting. ${ }^{22}$

Another decisive factor is the number of samples collected. The inappropriate collection plays a major role in false positive reporting. These inaccurate reports become a problem for patients about irrational use of antibiotics which may lead to an increase in hospital stay and cost of treatment unnecessary treatment and increased antibiotic resistance. 5

There should be a consistency between health knowledge and health practices, it is the cornerstone for the success of any disease prevention program. Dedicated and multidirectional efforts must be adopted to rectify this attitude and behaviour.

Our study has some limitations wherein the results are based on the answers given by health workers. For accurate results they should be observed and monitored.

\section{CONCLUSIONS}

Proper sample collection and transportation is a key step to patient care and is very useful for correct diagnosis. Most of the errors happen in the pre-analytical phase. Analytical and post-analytical errors are less frequent. In our study, we observed that the knowledge of participants improved significantly after continuous training sessions. This indicates that such a type of knowledge session is essential for improving sample collection practices. It is therefore important to train HCWs, who form the root of the health care system at regular intervals, to enhance knowledge significantly.

Data sharing statement provided by the authors is available with the full text of this article at jemds.com.

Financial or other competing interests: None.

Disclosure forms provided by the authors are available with the full text of this article at jemds.com.

\section{REFERENCES}

[1] Baron EJ, Peterson LR, Finegold SM. Selection, collection, and transport of specimens for microbial examination. Bailey and Scott's diagnostic microbiology. 9th edn. St. Louis Mosby-year Book 1994:53-64.

[2] Khan F, Sami H, Rizvi M, et al. Specimen collection: the art of laboratory science among the clinicians. J Patient Saf Infect Control 2017;5(1):35-9.

[3] Levin PD, Hersch M, Rudensky B, et al Routine surveillance blood cultures: their place in the management of critically ill patients. J Infect 1997;35(2):125-8.

[4] Nielsen J, Kolmos HJ, Rosdahl VT. Poor value of surveillance cultures for prediction of septicemia caused by coagulase-negative staphylococci in patients undergoing hemodialysis with central venous catheters. Scand J Infect Dis 1998;30(6):569-72.

[5] Kinirons B, Mimoz O, Lafendi L, et al. Chlorhexidine versus povidone iodine in preventing colonization of continuous epidural catheters in children: a randomized, controlled trial. Anesthesiology 2001;94(2):239-44.

[6] Mermel LA, Allon M, Bouza E, et al Clinical practice guidelines for the diagnosis and management of intravascular catheter-related infection: 2009 Update by the Infectious Diseases Society of America. Clin Infect dis 2009;49(1):1-45.

[7] Tenney JH, Reller LB, Mirrett S, et al Controlled evaluation of the volume of blood cultured in detection of bacteremia and fungemia J Clin Microbiol 1982;15(4): 558-61.

[8] Sandven P, Høiby EA The importance of blood volume cultured on detection of bacteraemia Acta Pathol Microbiol Scand B 1981;89(3):149-52.

[9] Chandak J, Loomba PS, Mishra B, et al. Impact of training on knowledge and practice of Nurses Regarding Hospital Infection Control in a tertiary care Hospital in Central. National Journal of Integrated Research of Medicine 2016;7(4):39-43.

[10] Ilstrup DM, Washington JA. The importance of volume of blood cultured in the detection of bacteremia and fungemia. Diagn Microbiol Infect Dis 1983;1(2):107-10.

[11] Gori E, Callea E, Alberani F, et al. Microbial monitoring and methods of sample collection: a GITMO survey (Gruppo Trapianto di Midollo Osseo) Ecancermedicalscience 2014;8:421.

[12] Calfee DP, Farr BM. Comparison of four antiseptic preparations for skin in the prevention of contamination of percutaneously drawn blood cultures: a randomized trial. J Clin Microbiol 2002;40(5):1660-5.

[13] Shafazand S, Weinacker AB. Blood cultures in the critical care unit: improving utilization and yield. Chest 2002;122(5):1727-36.

[14] Mythri H, Arun A, Kashinath KR. Perception and practice regarding infection control measures among healthcare workers in a tertiary center, South India. Journal of Ceramic Processing and Research 2015;7(2):109-14.

[15] Ojide CK, Onwuezobe IA, Asuquo EE, et al. Knowledge, attidude and practice of blood culture: a cross sectional study among medical doctors in a Nigerian tertiary hospital. Afr J Clin Exp microbio 2013;14(3):174-9.

[16] Jimenez CR, Koel-Simmilink M, Pham TV, et al. Endogenous peptide profiling of Cerebrospinal fluid by MALDI-TOF mass spectrometry optimization of magnetic bead-based peptide capture and analysis of pre-analytical variables. Proteomics Clin Appl 2007;1(11):1385-92.

[17] Levin PD, Hersch M, Rudensky B, et al Routine surveillance blood cultures: their place in the management of critically ill patients. J Infect 1997;35(2):125-8.

[18] Freifeld AG, Bow EJ, Sepkowitz KA, et al. Clinical practice guideline for the use of antimicrobial agents in neutropenic patients with cancer: 2010 update by the 
infectious diseases society of America. Clin Infect Dis 2011;52(4):56-93.

[19] Riley DK, Pavia AT, Beatty PG, et al. Surveillance cultures in bone marrow transplant recipients: worthwhile or wasteful? Bone Marrow Transpl 2000;15(3):469-73.

[20] Kokate SB, Rahangdale V, Katkar VJ. Impact of training on knowledge and practices of nurses regarding hospital infection control practices in a tertiary Care Hospital in Central India. Indian journal of applied science research 2017;7(8):233-34.
[21] Walsh TJ. Role of surveillance cultures in prevention and treatment of fungal infections Natl Cancer Inst Monogr 1990;9:43-5.

[22] Centers for Disease Control and Prevention; Infectious Disease Society of America; Guidelines for preventing opportunistic infections among hematopoietic stem cell transplant recipients. MMWR Rep 2000;49(RR-10):1125. 